\title{
Influence of growth on dust settling and migration in protoplanetary discs
}

\section{Elisabeth Crespe ${ }^{1}$, Jean-Francois Gonzalez ${ }^{1}$, Guillaume Laibe ${ }^{2}$, Sarah T. Maddison ${ }^{3}$ and Laure Fouchet ${ }^{4}$}

${ }^{1}$ Université de Lyon, Lyon, F-69003, France; Université Lyon 1, Villeurbanne, F-69622,

France; CNRS, UMR 5574, Centre de Recherche Astrophysique de Lyon; École normale supérieure de Lyon, 46, allée d'Italie, F-69364 Lyon cedex 07, France.

email: [elisabeth.crespe; jean-francois.gonzalez]@ens-lyon.fr

${ }^{2}$ Centre for Stellar and Planetary Astrophysics, School of Mathematical Sciences, Monash

University, Clayton Vic 3168, Australia.

${ }^{3}$ Centre for Astrophysics and Supercomputing, Swinburne University, PO Box 218, Hawthorn, VIC 3122, Australia.

${ }^{4}$ Physikalisches Institut, Universität Bern, CH-3012 Bern, Switzerland.

\begin{abstract}
To form meter-sized pre-planetesimals in protoplanetary discs, dust aggregates have to decouple from the gas at a distance far enough from the central star so they are not accreted. Dust grains are affected by gas drag, which results in a vertical settling towards the mid-plane, followed by radial migration. To have a better understanding of the influence of growth on the dust dynamics, we use a simple grain growth model to determine the dust distribution in observed discs. We implement a constant growth rate into a gas+dust hydrodynamics SPH code and vary the growh rate to study the resulting effect on dust distribution. The growth rate allows us to determine the relative importance between friction and growth. We show that depending on the growth rate, a range of dust distribution can result. For large enough growth rates, grains can decouple from the gas before being accreted onto the central star, thus contributing as planetary building rocks.
\end{abstract}

Keywords. planetary systems: protoplanetary disks, hydrodynamics, methods: numerical

\section{Hydrodynamics SPH Code}

We use a 3D, two-fluid (gas+dust) Smoothed Particle Hydrodynamics (SPH) code. The

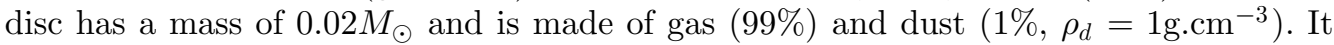
extends from $20 \mathrm{AU}$ to $400 \mathrm{AU}$ surrounding a $1 M_{\odot}$ star and is vertically isothermal. The initial temperature and density profiles scale as $T \propto r^{-3 / 4}$ and $\Sigma \propto r^{-3 / 2}$. The initial state contains 200,000 gas particles in a near-equilibrium disc that relaxes to a stationary state in approximately 8,000 years. Once the disc is relaxed, we add dust particles (size $\left.s_{0}=10 \mu \mathrm{m}\right)$ superimposed on the gas particles. The disc evolves over $10^{5}$ years.

\section{The dust growth model}

We implement a constant growth rate model for dust such that $S=S_{0}+\gamma \mathcal{T}$, where $S$ represent the grain size, $S_{0}$ its initial size, $\gamma$ is the growth rate and $\mathcal{T}$ represents the time (where capital quantities are dimensionless). The dimensionless growth rate can also be defined as the growth to friction rate:

$$
\gamma=\frac{\mathrm{d} S}{\mathrm{~d} \mathcal{T}}=\frac{\mathrm{d}\left(s / s_{\mathrm{opt}}\right)}{\mathrm{d}\left(t / t_{k}\right)}=\frac{\mathrm{d} s / \mathrm{d} t}{s_{\mathrm{opt}} / t_{k}}, \text { therefore } \begin{cases}\text { if } \gamma \gg 1, & \text { growth dominates, } \\ \text { if } \gamma \ll 1, & \text { friction dominates, }\end{cases}
$$

where $s_{\text {opt }}$ is an optimal size for migration and settling processes and $t_{k}$ is the orbital time. 


\section{Results}

Figure 1 shows the results obtained at the end of the simulation for different values of $\gamma$.

- Small values of $\gamma$ : grains are highly coupled to the turbulent gas and are distributed over the entire disc. The growth is not sufficient to change the regime of evolution. Grains migrate slowly toward the inner disc and can be accreted onto the central star.

- Intermediate values of $\gamma$ : grains grow slowly as they settle to the mid-plane where they experience a radial drift toward the inner part of the disc. As the growth is moderately efficient, grains can reach larger sizes. Their regime of evolution changes and they can evolve like large grains, decouple from the gas and move on keplerian orbits.

- Large values of $\gamma$ : growth is very efficient and grains do not have time to feel the gas, decoupling in less than one orbit. They experience little settling and radial drift and are distributed in the whole disc, as they were initially.

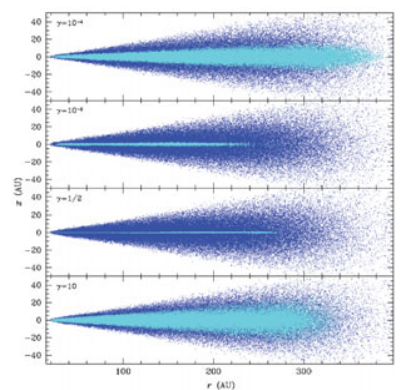

(a) Radial distribution in an edge-on view of the disc (dark=gas, light=dust) of SPH particles. From top to bottom: $\gamma=10^{-4}, 10^{-2}, 1 / 2$ and 10 .

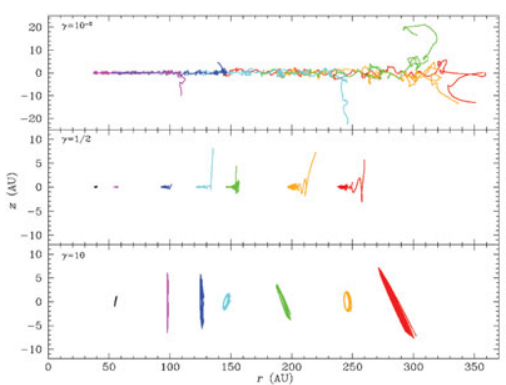

(b) Trajectories (in the $(r, z)$ plane) of SPH dust particles. From top to bottom: $\gamma=10^{-2}, 1 / 2$ and 10 .

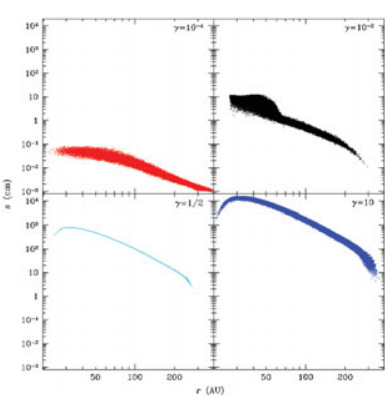

(c) Radial grains size distribution of SPH dust particles. From left to right and top to bottom: $\gamma=10^{-4}, 10^{-2}, 1 / 2$ and 10 .

Figure 1. Results of SPH simulations after $10^{5}$ years.

\section{Conclusion}

This study gives insight on grain size distributions in protoplanetary discs. The results obtained with $\gamma \approx 0.01$ are in good adequation with our study (Laibe et al. 2008) of grain growth using the model of Stepinski \& Valageas (1997). We conclude that the constant growth rate model gives an approximation of the physical growth process and underlines the fact that we can observe various dust distribution depending on the constant growth rate. SPH simulations validate the analytical results and direct numerical integration performed in Laibe et al. (2010a) for non-growing grains and generalized for growing grains in Laibe et al. (2010b). Acknowledgements: This research was partially supported by the Programme National de Physique Stellaire and the Programme National de Planétologie of CNRS/INSU, France, and the Agence Nationale de la Recherche (ANR) of France through contract ANR-07-BLAN-0221. STM acknowledges the support of a Swinburne Special Studies Program.

\section{References}

Barrière-Fouchet, L., Gonzalez, J.-F., Murray, J. R., Humble, R. J., \& Maddison, S. T. 2005, $A \& A, 443,185$

Laibe, G., Gonzalez, J.-F., Fouchet, L., \& Maddison, S. T. 2008, A\&A, 487, 265

Laibe, G., Gonzalez, J.-F., \& Maddison, S. T. 2010a, $A \mathscr{E} A$, under revision after referee's report Laibe, G., Gonzalez, J.-F., Maddison, S. T., Crespe, E., \& Fouchet, L. 2010b, A\&AA, submitted

Stepinski, T. F., \& Valageas, P., 1997, A\&AA, 319, 1007 